\title{
Fremdgasbehinderte und reine Molekularströmung durch eine vielfach geknidkte Röhre als Modell für ein Porensystem
}

\author{
Von J. W. Hiby \\ Forschungsinstitut Verfahrenstechnik ${ }^{1}$ an der Rhein.-Westf. Technischen Hochschule Aachen \\ und M. PaHL
}

Forschungsstelle für Spektroskopie in der Max-Planck-Gesellschaft, Hechingen

(Z. Naturforschg. 11 a, 80-84 [1956]; eingegangen am 24. August 1955)

Es wird eine Formel abgeleitet für die molekulare Gasströmung (mittlere freie Weglänge der Moleküle bezüglich gegenseitiger Zusammenstöße $\lambda \rightarrow \infty$ ) durch eine vielfach geknickte Röhre vom Durchmesser $2 a$. Neben den Wandstößen sollen noch wenige Zweierstöße mit schweren Fremdgasmolekülen (isotrop streuenden Zentren) auftreten können. Ist die mittlere freie Weglänge der strömenden Moleküle bezüglich der Stöße gegen Fremdgasmoleküle $\Lambda \gg$ die Länge $2 L$ eines geraden Rohrteilstückes, so läßt sich die fremdgasbehinderte Strömung als Funktion des Fremdgasdruckes $(\sim 2 a / \Lambda)$ bei beliebigem Parameter $L / a$ angeben. Bei gleichem Dichtegradienten $\mathrm{d} n / \mathrm{d} x$ folgt für das Verhältnis zwischen behinderter $\left(N_{\Lambda}\right)$ und reiner Molekularströmung $\left(N_{\Lambda_{\infty}}\right)$

$$
N_{\Lambda} / N_{\Lambda \infty}=1-\varkappa(2 a / \Lambda),
$$

worin $\varkappa$ eine Funktion von $L / a$ bedeutet. Für kurze Teilstücklängen $(2 \leqq L / a \leqq 10)$ werden errechnete Werte von $\varkappa$ mitgeteilt.

Den Fall von Röhren mit langen Teilstücken $(L \gg a)$ haben bereits $\mathrm{P}$ olla rd und P rese $\mathrm{nt}$ behandelt. Wir finden für $N_{\Lambda_{\infty}}$ exakt denselben Ausdruck. Hingegen unterscheidet sich unser Ergebnis für $N_{A}$ im Zahlenwert einer Konstanten. Die Diskrepanz wird geklärt.

Schließlich wird die rein molekulare Leitfähigkeit $\left(D_{0}\right)_{L}$ der vielfach geknickten Röhre verglichen mit derjenigen einer langen, geraden Röhre. Es ergibt sich ein Quotient

$$
\left(D_{0}\right)_{L /} /\left(D_{0}\right)_{L^{\prime} \rightarrow \infty}=f(L / a) \leqq 1,
$$

der für $L / a=3$ den Wert 0,8 annimmt. Dieses Ergebnis wirft ein Licht auf die rein molekulare Leitfähigkeit von unregelmäßigen Porensystemen, z. B. in einem Pulverhaufwerk; sie wird bekanntlich experimentell gefunden als lineare Extrapolation der P o i s e u i l l e - Geraden zum Mitteldruck Null und ist damit gegeben durch den Wert des Gleitungsgliedes $0,81 \quad\left(D_{0}\right) L^{\prime} \rightarrow \infty$. Dies führt zu der modellmäßigen Vorstellung eines Netzwerkes von Röhren mit $L / a \approx 3$.

Vor einiger Zeit haben $\mathrm{P}$ ollard und $\mathrm{Pre}$ sen $\mathrm{t}^{2}$ die Selbstdiffusion eines Gases in einer Röhre von kreisförmigem Querschnitt gaskinetisch nach dem Modell starrelastischer Kugeln ausführlich behandelt unter Berücksichtigung der Wirkung von Wand- und Zweierstößen. Für niedere Drucke liefern ihre Ergebnisse auch Aussagen über den Einfluß von Zweierstößen auf die molekulare Gasströmung durch Kreisröhren, sofern isotrope Richtungsverteilung der beim Zweierstoß gestreuten Teilchen vorausgesetzt werden kann. Dies ist der Fall, wenn Moleküle der Masse $m_{1}$ außer den Wandstößen nur seltene Zweierstöße gegen Teilchen der Masse $m_{2} \gg m_{1}$ ausführen, nicht aber, wenn $m_{1} \approx$ $m_{2}$ ist. Bei gleichen Massen der beiden Stoßpartner muß mit einer anisotropen Richtungsverteilung der

1 Träger: Forschungsgesellschaft Verfahrenstechnik e. V., Köln (GVT).

2 W. G. P ollard u. R. D. Present, Phys. Rev. (2) 73, 762 [1948]. gestreuten Teilchen gerechnet werden, die wir theoretisch abgeleitet ${ }^{3,4}$ und in die Berechnung der molekularen Strömung durch Widerstände verschiedener geometrischer Form eingeführt haben ${ }^{5,6}$. Im Zuge dieser Arbeiten sind von uns auch die molekularen Leitwerte $F$ für den Spaltkanal zwischen ebenen Flächen und für die Kreislochblende unter der Voraussetzung isotroper Streuverteilung neu berechnet worden als Funktion von $a / \Lambda$ ( $a$ : Lochradius, $A$ : mittlere freie Weglänge).

Der Vergleich experimenteller Messungen von $F(a / \Lambda)$ an porösen Medien mit theoretischen Ergebnissen führt dazu, als Modellbild ein Netzwerk von Lochblenden oder kurzen Röhren zu wählen ${ }^{6}$. Bei der Lochblende wirken sich nur Zweierstöße in naher Umgebung der Öffnung auf den molekularen

3 J. W. H i b y u. M. P a h l, Z. Phys. 129, 517 [1951].

4 J. W. H i b y u. M. P a h l, Z. Phys. 130, 348 [1951].

5 J.W. Hiby u. M. Pahl, Z. Naturforschg. 7 a, 533 [1952].

6 J.W.Hiby u. M. Pahl, Z. Naturforschg. 7 a, 542 [1952]. 
Leitwert aus. Die Behandlung der einzelnen kurzen Röhre wird bekanntlich dadurch erschwert, daß der Dichtegradient - im Gegensatz zur langen Röhre nicht konstant angenommen werden darf, sondern von der Mitte ab stetig über die Rohrenden hinaus bis in die angeschlossenen (großen) Gasvolumina hinein abnimmt. Bei einer vielfach geknickten Röhre aus kurzen, geraden Teilstücken entfällt jedoch diese Schwierigkeit, da hier, wegen des Fehlens hinreichend großer Volumina beiderseits eines Teilstücks, mit einem konstanten Dichtegradienten gerechnet werden kann.

In der vorliegenden Arbeit werden nun Rechnungen mitgeteilt, die die molekulare Strömung durch eine vielfach geknickte Kreisröhre behandeln. Zunächst wird der Einfluß von relativ seltenen Zweierstößen mit isotroper Verteilung der Streurichtungen berechnet (fremdgasbehinderte Molekularströmung). Dann machen wir den Grenzübergang zu verschwindendem Fremdgasdruck, also zur reinen Molekularströmung. Als wesentlicher Parameter erscheint jeweils das Verhältnis Länge zu Durchmesser der Teilstücke der geknickten Röhre. - Eine weitere Veröffentlichung wird die experimentelle Prüfung der Theorie der Fremdgasbehinderung und ihre Anwendung zur Bestimmung des Porendurchmessers von Glasfritten enthalten.

\section{Voraussetzungen}

Dem Ansatz der Rechnung liegen folgende Voraussetzungen zugrunde:

a) Längs der gesamten Röhre herrscht linearer Druckabfall.

b) Die geraden Teilstücke besitzen alle gleichen Radius $a$ und gleiche Länge $2 L$. Der Betrag der Strömung der leichten Moleküle durch den Quer. schnitt in der Mitte eines Teilstücks wird durch räumliche Integration über die aus allen Richtungen einfallenden Teilchen gewonnen. An Stelle der beliebig schrägen Begrenzung (Abb. 1) stehen offene Stirnflächen im Mittel beide senkrecht zur Rohrachse, wie in Abb. 2 wiedergegeben, die auch weitere Bezeichnungen enthält. Der Dichtegradient $\mathrm{d} n / \mathrm{d} x$ besitze auch in diesem stark idealisierten Modell denselben Wert wie in einem Rohrstück mit beliebig schrägen Enden von gleicher Länge $2 L$.

c) Für die mittlere freie Weglänge der leichten Moleküle untereinander soll gelten: $\lambda \rightarrow \infty$. d) Die mittlere freie Weglänge für Stöße der leichten Moleküle gegen die schweren (praktisch ruhenden) Fremdgasmoleküle sei überall

$$
\text { const }=A \gg L \text {. }
$$

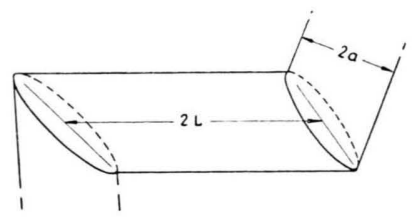

Abb. 1. Teilstück einer vielfach geknickten Röhre. Winkel Stoßebene-Achse beliebig.

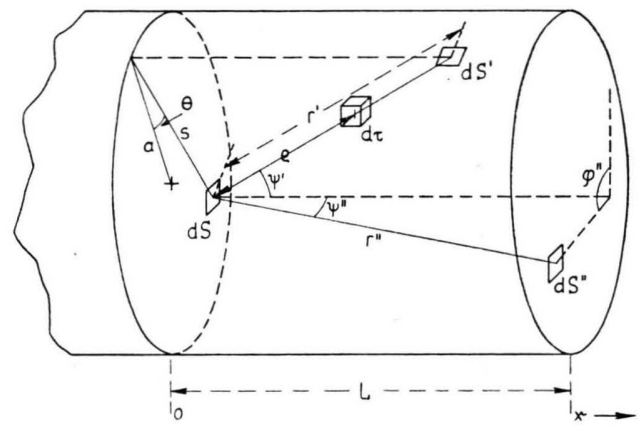

Abb. 2. Schema eines Rohrteilstückes mit Koordinatensystem und Bezeichnungen. Gesamtlänge $2 L$, Mitte bei $x=0$.

Die gesamte Strömung $N$ der leichten Moleküle durch den betrachteten Rohrquerschnitt bei $x=0$ setzt sich nun zusammen aus einem Anteil $N_{\mathrm{w}}$ von Molekülen, die geradewegs von der Wand her einfallen, und einem Anteil $N_{\mathrm{g}}$ von solchen, die von einem Zweierstoß mit einem schweren Molekül kommen. Bezüglich der $N_{\mathrm{w}}$-Teilchen wird für die Zwecke der Integration noch unterschieden zwischen den Stößen an der Zylinderwand $\left(N_{\mathrm{w}_{1}}\right)$ und der von den Stirnflächen kommenden Strömung $\left(N_{\mathrm{w}_{2}}\right)$ :

$$
N=N_{\mathrm{w}}+N_{\mathrm{g}}=N_{\mathrm{w}_{1}}+N_{\mathrm{w}_{2}}+N_{\mathrm{g}}[\text { Moleküle/sec }] .
$$

\section{Fremdgasbehinderte Molekularströmung}

Die einzelnen Anteile der Strömung ergeben sich nun folgendermaßen:

a) Für die vom Zylindermantel ausgehenden Teilchen gilt nach $\mathrm{P}$ oll ard und $\mathrm{Presen} \mathrm{t}{ }^{2}$, Gl. (24)

$$
N_{\mathrm{w}_{1}}=-\bar{v} \frac{\mathrm{d} n}{\mathrm{~d} x} \iint \mathrm{d} S \int_{\psi_{0}}^{\pi / 2} s \cos ^{2} \psi^{\prime} \cdot e^{-\bar{s} \sin \psi^{\prime}} \mathrm{d} \psi^{\prime} .
$$

Hierin bedeuten: $\bar{v}$ die mittlere Teilchengeschwindigkeit und $\psi_{0}$ den Polabstand für die Randpunkte des 
Zylindermantels. Über das Azimut $\varphi$ ist bereits integriert. Bezüglich der verwendeten Bezeichnungen verweisen wir auf Abb. 2, hinsichtlich der Ableitungen der Gln. (1), (2) und (3) auf die zitierte Arbeit von Pollard und Present.

b) Die von den Stirnflächen kommende Strömung beträgt nach analoger Überlegung

$N_{\mathrm{w}_{2}}=-L v \frac{\mathrm{d} n}{\mathrm{~d} x} \iint \mathrm{d} S \int_{0}^{\psi_{0}} \sin \psi^{\prime \prime} \cos \psi^{\prime \prime} \cdot e^{-\frac{L}{\Lambda \cos \psi^{\prime \prime}} \mathrm{d} \psi^{\prime \prime}}$,

wobei wiederum wie in (1) die $e$-Funktion im Integranden die Abnahme des Strömungsanteils mit wachsendem Fremdgasdruck (kleiner werdendem $A$ ) beschreibt.

c) Man gelangt weiterhin zu dem Strömungsanteil der von Zweierstößen in sämtlichen Volumelementen $\mathrm{d} \tau$ kommenden Moleküle

$$
\begin{aligned}
& N_{\mathrm{g}}=-\frac{\bar{v}}{2 \Lambda} \frac{\mathrm{d} n}{\mathrm{~d} x} \iint \mathrm{d} S \\
& \cdot\left[L^{2} \int_{0}^{\psi_{0}} \sin \psi^{\prime \prime} \mathrm{d} \psi^{\prime \prime}+s^{2} \int_{\psi_{0}}^{\pi / 2} \frac{\cos ^{2} \psi^{\prime}}{\sin \psi^{\prime}} \mathrm{d} \psi^{\prime}\right] .
\end{aligned}
$$

Pollard und Present erzielen die weiteren Integrationen durch Beschränkung auf den Fall langer Röhren: $L \gg a, \Lambda$ beliebig. Wir beschränken uns hingegen auf eine relativ geringe Anzahl von isotrop streuenden Zweierstößen, setzen also $A \gg L$ und können dafür $L / a$ beliebig wählen. Diese Festsetzung erlaubt nun, die Entwicklung der Exponentialfunktion bei $N_{\mathrm{w}_{1}}$ und $N_{\mathrm{w}_{2}}$ bereits nach dem ersten Gliede abzubrechen. (Eine Näherung gleicher Ordnung wurde auch bei der Ableitung von Gl. (3) verwendet.)

Nach Integration über $\psi$ und Normierung des Flächenintegrals auf die unabhängigen Variablen $s$ und $\Theta$ [Gl. (16) bei Pollard und Present] erhält man als Näherung von erster Ordnung in $L / A$ :

$$
\begin{aligned}
& N=\frac{\bar{v}}{2} a^{2} \pi \frac{L^{2}}{\Lambda} \frac{\mathrm{d} n}{\mathrm{~d} x}--\frac{\bar{v}}{2} a \frac{\mathrm{d} n}{\mathrm{~d} x} \int_{-\pi / 2}^{+\pi / 2} \cos \Theta \mathrm{d} \Theta \\
& \cdot \int_{0}^{2 a \cos \theta} \mathrm{d} s\left[\frac{\pi}{2} s-s \operatorname{arctg} \frac{s}{L}+\frac{L}{\Lambda} \sqrt{L^{2}+s^{2}}+\frac{s^{2}}{L} \ln \frac{s}{L+\sqrt{L^{2}+s^{2}}}\right] .
\end{aligned}
$$

Integration über $s$ und $\Theta$ liefert nach längerer Rechnung Gl. (5) :

$$
\begin{gathered}
N=-\frac{2 \pi}{3} \bar{v} a^{3} \frac{\mathrm{d} n}{\mathrm{~d} x}\left\{1+\frac{3 B}{4}-\frac{3 B^{2}}{\pi} I_{1}-\frac{3}{\pi} I_{2}\right. \\
\left.-\frac{a}{\Lambda}\left[3 B^{2}-\frac{4 B^{2}}{\pi} I_{3}-\frac{8 B^{3}}{\pi} I_{4}-\frac{4}{\pi}\left(I_{5}-I_{6}\right)\right]\right\} \\
\text { mit } \quad B=L / 2 a .
\end{gathered}
$$

Die Integrale $I_{1} \ldots I_{6}$ und ihre Lösungen lauten:

$$
I_{1}=\int_{0}^{\pi / 2} \cos \Theta \cdot \operatorname{arctg}\left(B^{-1} \cos \Theta\right) \mathrm{d} \Theta .
$$

Substitution $x=B^{2}+\cos ^{2} \Theta$ und eine Produkt-Integration führen zur Lösung:

$$
I_{1}=\frac{\pi}{2}\left(\sqrt{1+B^{2}}-B\right) .
$$

Es ist $\quad I_{2}=\int_{0}^{\pi / 2} \cos ^{3} \Theta \cdot \operatorname{arctg}\left(B^{-1} \cos \Theta\right) \mathrm{d} \Theta$.

Hier gelangt man auf ähnlichem Wege zu

$$
\begin{gathered}
I_{2}=\frac{\pi}{6}\left(-\frac{3}{2} B+B^{3}+2 \sqrt{1+B^{2}}-B^{2} \sqrt{1+B^{2}}\right) . \\
I_{3}=\int_{0}^{\pi / 2} \cos ^{2} \Theta \cdot \sqrt{1+B^{-2} \cos ^{2} \Theta} \mathrm{d} \Theta \quad \text { führt zu } \\
I_{3}=-\frac{1}{3} B \sqrt{1+B^{2}} K(\alpha)+\frac{2+B^{2}}{3 B} \sqrt{1+B^{2}} E(\alpha) ;
\end{gathered}
$$

darin bedeuten $K(\alpha)$ und $E(\alpha)$ die vollständigen elliptischen Integrale 1. und 2. Gattung, $\alpha=\arcsin$ $\left(1 / \sqrt{1+B^{2}}\right)$.

Es ist

$$
I_{4}=\int_{0}^{\pi / 2} \cos \Theta \cdot \ln \left(B^{-1} \cos \Theta+\sqrt{1+B^{-2} \cos ^{2} \Theta}\right) \mathrm{d} \Theta .
$$

Nach Substitution $x=B^{-1} \cos \Theta$ und Produkt-Integration findet man die Lösung

$$
\begin{gathered}
I_{4}=\sqrt{1+B^{2}}[K(\alpha)-E(\alpha)] . \\
I_{5}=\int_{0}^{\pi / 2} \cos ^{4} \Theta \cdot \ln \left(B^{-1} \cos \Theta\right) \mathrm{d} \Theta
\end{gathered}
$$

$\operatorname{ergibt}^{7} \quad I_{5}=\frac{7 \pi}{64}-\frac{3 \pi}{16} \ln (2 B)$.

$$
I_{6}=\int_{0}^{\pi / 2} \cos ^{4} \Theta \cdot \ln \left(1+\sqrt{1+B^{-2} \cos ^{2} \Theta}\right) \mathrm{d} \Theta
$$

wurde graphisch für mehrere Werte von $B$ zwischen 1 und $\infty$ gelöst und läßt sich mit großer Genauigkeit darstellen als

$$
I_{6}=\frac{3 \pi}{16} \ln 2+0,09 B^{-2} .
$$

7 Vgl. z. B. D. Bierens de Haan, Tables d'Intégrales définies, Pag. 432, Table 331, Nr. 5. 
Nach Einsetzen der Integrale erhält man schließlich für die Strömung durch den Rohrquerschnitt als Näherung von 1 . Ordnung in $L / \Lambda$ den Ausdruck $N_{\Lambda}$ (für $\Lambda \gg L$ ) bzw. den Grenzwert $N_{\Lambda \infty}$ (für $\Lambda \rightarrow \infty)$ :

$$
\begin{aligned}
& N_{.1}= N_{A_{\infty}}+\frac{\pi}{3} v a^{3} \frac{\mathrm{d} n}{\mathrm{~d} x} \frac{2 a}{A} \\
& \cdot\left[\frac{3}{2} \ln 2-\frac{7}{16}+0,1146 B^{-2}+\frac{3}{4} \ln B+3 B^{2}\right. \\
&\left.-\frac{20}{3 \pi} B^{3} \sqrt{1+B^{2}}(K-E)-\frac{8}{3 \pi} B \sqrt{1+B^{2}} \cdot E\right], \\
& N_{\Lambda_{\infty}=}-\frac{2 \pi}{3} \bar{v} a^{3} \frac{\mathrm{d} n}{\mathrm{~d} x} \\
& \cdot\left(1+\frac{3}{2} B-\sqrt{1+B^{2}}-B^{2} \sqrt{1+B^{2}}+B^{3}\right)
\end{aligned}
$$

mit $B=L / 2 a$.

Das Verhältnis zwischen der behinderten und der reinen Molekularströmung bei gleichem Dichtegradienten $\mathrm{d} n / \mathrm{d} x$ folgt daraus zu

$$
\frac{N_{\Lambda}}{N_{\Lambda \infty}}=1-\varkappa \frac{2 a}{\Lambda}
$$

hierin ist $\varkappa$ eine Funktion von

$$
2 B=2 L / 2 a=\text { Rohrstücklänge/Durchmesser, }
$$

die z. B. folgende Werte annimmt:

\begin{tabular}{|c|ccccc|}
\hline$L / a$ & 2 & 3 & 4 & 5 & 10 \\
\hline$\varkappa$ & 0,436 & 0,540 & 0,620 & 0,695 & 0,885 \\
\hline
\end{tabular}

Für lange Rohrteilstücke $(L \gg a)$ erhalten wir durch Reihenentwicklung von (6a) als Näherung von 1 . Ordnung in $a / L$ :

$$
\frac{N_{\Lambda}}{N_{\Lambda_{\infty}}}=1-\frac{0,3104+0,75 \ln (L / 2 a)}{1-0,75 a / L} \frac{a}{A} ; \quad \Lambda \gg L \gg a \text {. }
$$

An Stelle der Konstanten 0,3104 geben $\mathrm{P}$ o 11 a r d und Prese $n t$ den Wert 0,4764 an in ihrer Gl. (32), die sonst mit unserem Ausdruck (8) übereinstimmt. Wir können zeigen, daß unser Ausdruck die bessere Näherung darstellt. P o l l a r d und Present nehmen zunächst den Grenzübergang $L \gg a$ vor, approximieren also mit ihrer Gl. (29) die Funktion $N(a / \Lambda, a / L)$ in einem schmalen Streifen der $a / \Lambda-a / L$ Ebene, der an die $a / \Lambda$-Koordinatenachse angrenzt. Wir gehen dagegen von der Voraussetzung $A \gg L \gg a$ aus, so daß unsere Approximation (6a) sich auf einen Streifen bezieht, der der $a / L$-Koordinatenachse anliegt. Die Funktion $N$ ist im Ursprung $a / \Lambda=a / L=0$ nicht differenzierbar, da in diesem Punkt die partielle Ableitung nach $a / \Lambda$ unendlich wird. Infolgedes-

8 Die rein molekulare Leitfähigkeit der geknickten Röhre kann natürlich auch direkt aus den Gln. (1) und (2) unter Verwendung des Grenzüberganges $\Lambda / L \rightarrow \infty$ berechnet wer- sen strebt bei Annäherung an den Ursprung die Streifenbreite, die einer bestimmten Güte der genannten Approximationen entspricht, gegen Null; die beiden Streifen laufen spitz zu und überdecken sich gegenseitig nicht. Die Bedingung $\Lambda \gg L \gg a$ bezieht sich auf Punkte in unserem Streifen, so daß lediglich unsere Gl. (8) als Näherung brauchbar ist.

Für die reine Molekularströmung durch lange Rohrteilstücke $(L \gg a, \Lambda \rightarrow \infty)$ folgt aus $(6 \mathrm{~b})$ :

$$
N_{\Lambda_{\infty}}=-\frac{2 \pi}{3} \bar{v} a^{3} \frac{\mathrm{d} n}{\mathrm{~d} x}\left(1-\frac{3}{4} \frac{a}{L}\right)
$$

in exakter Übereinstimmung mit Gl. (33) von Pollard und Present.

\section{Reine Molekularströmung}

Der rein molekulare Leitwert einer langen, geraden Röhre ist nach $\mathrm{Knudsen}$

$$
F_{0}=\frac{2 \pi}{3} \frac{\bar{v} a^{3}}{L} \text {. }
$$

Bezogen auf die Längen- und Querschnittseinheit der Röhre ergibt sich eine molekulare „Leitfähigkeit“

$$
D_{0}=\frac{2}{3} \bar{v} a
$$

mit der Dimension eines Diffusionskoeffizienten. Aus (6b) und (9) folgt $^{8}$ für das Verhältnis der rein molekularen Leitfähigkeit der geknickten Röhre zu der einer langen, geraden Röhre gleichen Durchmessers ein Wert $\leqq 1$, der eine Funktion des Parameters $L / a$ ist und z. B. folgende Zahlenwerte annimmt:

\begin{tabular}{|l|ccccc|}
\hline$L / a$ & 2 & 3 & 4 & 5 & 10 \\
\hline$\left(D_{0}\right)_{L /\left(D_{0}\right)_{L^{\prime} \rightarrow \infty}}$ & 0,672 & 0,766 & 0,820 & 0,855 & 0,925 \\
\hline $0,98\left(D_{0}\right)_{L} /{ }^{1}\left(D_{0}\right)_{L^{\prime} \rightarrow \infty}$ & 0,699 & 0,797 & 0,853 & 0,889 & 0,962 \\
\hline
\end{tabular}

In der letzten Zeile ist noch eine Korrektur vorgenommen zur Berücksichtigung einer geringfügigen Abweichung der Wandreflexion vom Kosinusgesetz. Für die wichtigsten Gase kann nämlich der $M$ ax wellsche Koeffizient für den Anteil der diffusen Reflexion $f \approx 0,98$ gesetzt werden ${ }^{5}$ (links hochgestellter Index). Die Leitfähigkeit vergrößert sich dann um den Faktor $(2-f) / f \approx 1,04$. In der letzten Zeile der Tabelle wird somit angegeben, um welchen Faktor die molekulare Leitfähigkeit einer geknickten Röhre bei realer Wandreflexion herab-

den, ohne den Umweg der Berücksichtigung von Zweierstößen. 
gesetzt ist im Vergleich zur Leitfähigkeit der langen, geraden Röhre [Gl. (11)], abgeleitet für ideal diffuse Wandreflexion.

Unser Ergebnis vermag eine Erklärung zu geben für den Grenzwert der molekularen Leitfähigkeit, wie er experimentell bei der Durchströmung von feinkörnigen Haufwerken gefunden wird. Läßt man ein Gas bei verschiedenen, mittleren Drucken laminar durch eine pulvrige Substanz strömen, dann findet man bekanntlich, daß die Leitfähigkeit entsprechend der Gleichung von $\mathrm{P}$ o is e uille linear mit dem Mitteldruck abnimmt. Bei Druckerniedrigung bis in den molekularen Bereich durchläuft die Leitfähigkeit jedoch nicht - wie bei der geraden Kapillare ein Minimum, sondern fällt im allgemeinen mit unverändertem Gradienten weiter linear ab bis zum molekularen Grenzwert. Dieser Grenzwert wird nach dem experimentellen Befund also gegeben durch das konstante Gleitungsglied $0,81{ }^{1}{ }^{1}\left(D_{0}\right)_{L^{\prime} \rightarrow \infty}$, das bekanntlich bei Gasen zur P o i s e uille schen Gleichung hinzutritt ${ }^{9} .{ }^{1}\left(D_{0}\right)_{L^{\prime} \rightarrow \infty}$ ist die molekulare Leitfähigkeit eines Modellsystems von geraden, parallelgeschalteten Kapillaren (mit diffus reflektierenden Wänden), das im laminaren Bereich das untersuchte, unregelmäßige Porensystem hinsichtlich der Leitfähigkeit ersetzen würde.

Unser Rechnungsergebnis zeigt nun, daß die Herabsetzung der molekularen Grenzleitfähigkeit um den Faktor 0,81 gedeutet werden kann als „Knickbehinderung“ in einem Modellsystem geknickter Kapillaren, für deren gerade Teilstücke das Verhältnis Länge zu Durchmesser $\approx 3$ beträgt. Wir haben bereits früher ${ }^{6}$ gezeigt, daß der Gradient des Anstieges der Leitfähigkeit als Funktion des Mittel-

9 Vgl. z. B. S. Dushman, Scientific Foundations of Vacuum Technique, New York 1949, S. 112.

10 E. M a n e g o ld, Kapillarsysteme Bd. I, S. $360 \mathrm{ff}$. Straßenbau, Chemie u. Technik Verlag GmbH, Heidelberg druckes sich ebenfalls richtig ergibt (etwa gleicher Anstieg im molekularen und laminaren Bereich), wenn man das genannte Modell mit $L / a \approx 3,5$ verwendet.

Eine weitere Stütze für unser Modell liefert auch eine geometrische Betrachtung an dichtesten Kugelpackungen. Die kubische 12er-Packung von gleichgroßen Kugeln des Radius $r$ ist durchzogen von einem System netzartig sich überschneidender, gerader Kanalstücke von dreiecksähnlichem Querschnitt. Manegold ${ }^{\mathbf{1 0}}$ berechnet die Länge $2 \mathrm{~L}$ und den mittleren Querschnitt der geraden Kanal-

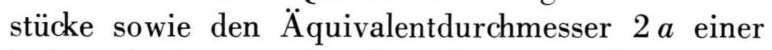
Röhre gleichen, runden Querschnittes: $2 L=2,45 r$, $2 a=0,736 r$, demnach $L / a=3,3$. Bei einer hexagonalen 12er-Packung tritt allerdings auch eine Sorte von langen, durchgehenden Kanälen auf, die aber in unregelmäßigen Kugelschüttungen natürlich häufig unterbrochen werden. In der unregelmäßigen Schüttung wird man im Gegenteil eher eine gewisse Verringerung des Quotienten $L / a$ gegenüber der regelmäßigen Packung erwarten. Es sprechen also auch geometrische Gründe dafür, daß man die Hohlräume in einer Schüttung kugelähnlicher Körner im Hinblick auf die Durchströmung durch ein Modell geknickter Röhren mit $L / a \approx 3$ ersetzen darf. Freilich wird dieser Wert des Parameters nicht allgemein gültig sein; die individuelle Struktur eines Haufwerkes kann zu Abweichungen nach oben oder unten führen, die experimentell noch nicht genau untersucht worden sind. Die zahlenmäßige Übereinstimmung von Gleitungsglied und molekularem Grenzwert ist durchaus unerwartet und kann nicht anders als zufällig genannt werden.

1955. - Man erkennt das (auch bei der hexagonalen Pakkung auftretende) Kanalstück auf Abb. 228, Streckenzug B-D, über D hinaus um die gleiche Strecke verlängert. 